Open Access

\title{
The burden of bacteremia and invasive diseases in children aged less than five years with fever in Italy
}

Chiara Azzari ${ }^{1 *}$, Maria Moriondo ${ }^{1}$, Pasquale Di Pietro ${ }^{2}$, Cesare Di Bari ${ }^{3}$, Massimo Resti ${ }^{4}$, Francesco Mannelli ${ }^{4}$, Susanna Esposito ${ }^{5}$, Guido Castelli-Gattinara ${ }^{6}$, Antonio Campa ${ }^{7}$, Fernando Maria de Benedictis ${ }^{8}$, Gianni Bona ${ }^{9}$, Lisa Comarella ${ }^{10}$, Katsiaryna Holl ${ }^{11}$ and Federico Marchetti ${ }^{12}$

\begin{abstract}
Background: Invasive diseases (ID) caused by Streptococcus pneumoniae (S. pneumoniae), Haemophilus influenzae (H. influenzae), and Neisseria meningitidis are a major public health problem worldwide. Comprehensive data on the burden of bacteremia and ID in Italy, including data based on molecular techniques, are needed.

Methods: We conducted a prospective, multi-centre, hospital-based study (GSK study identifier: 111334) to assess the burden of bacteremia and ID among children less than five years old with a fever of $39^{\circ} \mathrm{C}$ or greater. Study participation involved a single medical examination, collection of blood for polymerase chain reaction (PCR) and blood culture, and collection of an oropharyngeal swab for colonization analysis by PCR.

Results: Between May 2008 and June 2009, 4536 patients were screened, 944 were selected and 920 were enrolled in the study. There were 225 clinical diagnoses of ID, $9.8 \%$ (22) of which were bacteremic. A diagnosis of sepsis was made for 38 cases, 5.3 \% (2) of which were bacteremic. Among the 629 non-ID diagnoses, $1.6 \%$ (10) were bacteremic. Among the 34 bacteremic cases, the most common diagnoses were community-acquired pneumonia (15/34), pleural effusion (4/34) and meningitis (4/34). S. pneumoniae was the most frequently detected bacteria among bacteremic cases (29/34) followed by H. influenzae (3/34). Ninety percent (27/30) of bacteremic patients with oropharyngeal swab results were colonized with the studied bacterial pathogens compared to $46.1 \%(402 / 872)$ of non-bacteremic cases $(p$ $<0.001)$. PCV7 (7-valent pneumococcal conjugate vaccine) vaccination was reported for $55.9 \%$ (19/34) of bacteremic cases. S. pneumoniae serotypes were non-vaccine serotypes in children who had been vaccinated. Mean duration of hospitalization was longer for bacteremic cases versus non-bacteremic cases (13.6 versus 5.8 days).
\end{abstract}

Conclusions: These results confirm that $S$. pneumoniae is one of the pathogens frequently responsible for invasive disease.

Keywords: Fever, Invasive disease, Bacteremia, Streptococcus pneumoniae, Haemophilus influenzae, Pneumococcal vaccine

\footnotetext{
*Correspondence: Chiara.azzari@unifi.it

'Division of Pediatric Immunology, Department of Health Sciences, University of Florence and Anna Meyer Children's University Hospital, Florence, Italy Full list of author information is available at the end of the article
} 


\section{Background}

Invasive diseases (ID) are considered any disease where microorganisms are identified in the normally sterile body fluids. Bacteremia may be due to predisposing risk factors (sickle cell anemia, oncologic disease, immunodeficiency, indwelling central catheter, etc.), definitive focal infection (pneumonia, meningitis, cellulites, etc.) or present as occult bacteremia, i.e. the presence of pathogenic bacteria in the blood of a well-appearing febrile child without an identifiable focus of infection. Invasive diseases (ID) caused by Streptococcus pneumoniae (S. pneumoniae), Haemophilus influenzae (H. influenzae), and Neisseria meningitidis including pneumonia, bacteremia and meningitis and other illnesses, are a major public health problem worldwide. In Europe, estimated annual invasive pneumococcal disease (IPD) incidence per 100,000 population has been reported in hospital-based studies to range from 0.3 to 20.3 , and varies by age, with the highest incidence observed in children less than two years of age [1]. Availability of pneumococcal vaccines has led to substantial decreases in overall IPD in the United States and Europe [2-4], and there is also evidence of benefits for the unvaccinated population through herd immunity [5]. Concerns about potential replacement of vaccine serotypes with non-vaccine serotypes still need to be addressed.

Knowledge of Italian epidemiologic features of bacteremia and ID relies on voluntary reporting and sending strains to the national reference laboratory, therefore, the true burden may be underestimated and under-reported [6-8]. More comprehensive surveillance, supported by molecular epidemiological analyses, is considered a priority among all the European countries by the European Centre for Disease Control and Prevention.

We conducted a prospective, multi-centre, hospitalbased study to assess the burden of bacteremia and ID, including cost of hospitalization, clinical severity, causative agents and vaccination status, among children less than five years of age with a fever of $39{ }^{\circ} \mathrm{C}$ or greater. Additionally, oropharyngeal colonization by the investigated bacteria and the estimated clinical expenses due to bacteremia were examined.

\section{Methods}

\section{Study objectives and definitions}

The primary objective of the study was to assess the incidence of bacteremia and invasive diseases (ID) in children $<5$ years of age in pediatric hospitals in Italy. Secondary objectives were to determine the clinical severity of bacteremia and describe concomitant contributing clinical factors; to determine the rate of hospitalization due to bacteremia; to identify the causative organisms of bacteremia with a major interest in S. pneumoniae, H. influenzae and Neisseria meningitidis and their susceptibility to antimicrobial agents; to assess the rate of IPD in patients previously vaccinated with pneumococcal vaccine; to assess polymerase chain reaction (PCR) sensitivity in diagnosis of bacteremia; to assess biological samples positive cultures or PCR according to subject's previous antibacterial treatment; to assess oropharyngeal colonization by the investigated bacteria; to assess the incidence of febrile illness in children $<5$ years of age; and to estimate clinical management expenses due to bacteremia.

Children were enrolled in this prospective, hospitalbased study (GSK study identifier: 111334) at emergency departments or inpatient clinics at participating pediatric hospitals in Genova, Bari, Napoli, Roma, Firenze, Trieste, Ancona, Novara and Milano from May 2008 to June 2009.

\section{Sample size}

The expected number of cases of children with fever of at least $39{ }^{\circ} \mathrm{C}$ was estimated based on the hospital activity reports over the previous three years. Sample size was derived from the figures given by each participating centre on the at-risk population (i.e. children $<5$ years of age with fever $\geq 39^{\circ} \mathrm{C}$ ) attending their hospitals in a 12 month timeframe. Assuming compliance to the study requirements (i.e. informed consent of both parents, invasive procedures, etc.) of $33 \%$ from each centre, a mean number of 400 subjects per centre were expected for a total of nearly 4000 enrolled subjects.

\section{Inclusion and exclusion criteria}

Children were eligible if they were less than five years of age, had a fever of at least $39{ }^{\circ} \mathrm{C}$ at enrolment or at home with at least one measurement in the $12 \mathrm{~h}$ preceding enrolment, and had been a resident in the district of the hospital for at least the last three months. At home, fever may have been measured by parents using any method; at enrolment fever was measured for infants under four weeks using an electronic thermometer in the axilla, and for children four weeks to five years by an electronic thermometer or chemical dot thermometer in the axilla or an infrared tympanic thermometer. Children with a presumptive diagnosis of gastroenteritis or a clinical diagnosis of infectious exantherma were excluded from the study. Informed consent was obtained from each parent/guardian prior to performance of any studyspecific procedures.

\section{Study recruitment and procedures}

The study involved a single visit which included a medical examination, collection of a blood sample for local biochemistry and bacteriology (culture), and collection of a blood and oropharyngeal sample for invasive disease or colonization to be sent for PCR to the study laboratory. Data on demographics, medical history, vaccination status and previous antibiotic treatment and current fever 
treatment were also collected at the visit. A log book with aggregated number of fever and ID cases presenting to the centre, regardless of participation status in the study, was maintained at each study centre.

Bacteremic cases were defined as subjects fulfilling inclusion and exclusion criteria with a blood sample positive for bacteria either by PCR or by blood culture. A case was considered non-bacteremic if the PCR or blood culture testing was negative for the 3 bacteria that were tested. Bacteremia predisposing factors included sickle cell anemia, oncologic disease, immunodeficiency, indwelling central catheter or shunts, agranulocitosis, aplastic anemia, arteritis, renal transplant, congenital heart abnormalities, congestive heart failure, cystic fibrosis, human immunodeficiency virus (HIV), Lyme disease, Kawasaki disease and nephritic syndrome.

Diagnoses of focal infections such as communityacquired pneumonia (CAP), pleural effusion, meningitis, cellulites, lymphadenitis, otitis media, or pharyngitis were made on the basis of available diagnostic findings (such as $\mathrm{X}$-rays or cerebrospinal fluid findings). The same criterion was applied for systemic infections (sepsis). A patient could receive more than one diagnosis. The clinical diagnosis made by the investigator of community-acquired was categorized as either a diagnosis of invasive disease or as a diagnosis of non-invasive disease. Pneumonia, pleural effusion, meningitis and sepsis were considered primary clinical diagnoses suggestive of invasive disease. Although sepsis was considered invasive, it was also examined in a separate category. Other diagnoses such as cellulites, lymphadenitis, otitis media and pharyngitis were considered non-invasive.

\section{Laboratory analysis}

The presence of S. pneumoniae, Neisseiria meningitidis or $H$. influenzae type b (Hib) DNA in biological samples and isolates was evaluated by Realtime PCR (RT-PCR) using the EuSep screen kit (Eurospital, Trieste, Italy) according to manufacturer instructions. The cycle threshold $\left(C_{T}\right)$ value is the PCR cycle number (out of 45$)$ at which the measured fluorescent signal exceeds a calculated background threshold identifying amplification of the target sequence. If no increase in fluorescent signal is observed after 45 cycles, the sample is assumed to be negative. The presence of Haemophilus different from Hib was evaluated by a home-made method, using bexA and ompP2 genes as targets and previously published primers and probes (REfX). The presence of S. pneumoniae DNA was confirmed by the amplification of CpsA gene in end-point PCR as previously reported $[9,10]$ and briefly described in the next paragraph. Only samples positive for both lytA gene in RT-PCR and CpsA in end-point PCR were included in pneumococcal serotyping analysis.

For pneumococcal serotyping, RT-PCR was performed in $25 \mu \mathrm{L}$ reaction volumes containing $2 \mathrm{x}$ TaqMan
Universal Master Mix (Applied Biosystem, Foster City, CA, USA); primers and JOE labeled probes were used at a concentration of $400 \mathrm{nM}$; FAM labeled probes at a concentration of $200 \mathrm{nM}$. Six $\mu \mathrm{l}$ of DNA extract was used for each reaction. All reactions were performed in triplicate. Negative controls (both blood samples from healthy controls and sterile water samples) and positive controls (blood samples or cerebrospinal fluid samples known to be positive for S. pneumoniae both by cultural and molecular methods) were included in every run. DNA was amplified in an $A B I 7500$ sequence detection system (Applied Biosystem, Foster City, CA, USA) using, for all the primers couples, the same cycling parameters as follows: $50{ }^{\circ} \mathrm{C}$ for two minutes for UNG digestion, $95{ }^{\circ} \mathrm{C}$ for $10 \mathrm{~min}$ followed by 45 cycles of a two-stage temperature profile of $95^{\circ} \mathrm{C}$ for $15 \mathrm{~s}$ and $60^{\circ} \mathrm{C}$ for one minute. If no increase in fluorescent signal was observed after 45 cycles for any of the primer/probe set, the sample was assumed to be negative with the serotype specific primers and was reported as non-typeable.

For culture purposes, 4-6 ml of blood samples (up to three sets) were taken at the fever peak and immediately sent to the local laboratory; procedures established by each hospital for these tests were used, as described previously [11].

\section{Health economics}

Economic data relevant to expenses due to bacteremia were also collected from a subset of children in order to examine and estimate the associated clinical management expenses.

\section{Statistical analysis}

Demographic and clinical characteristics were tabulated by means of descriptive statistics. Medical history, other relevant clinical information and PCR results were presented by means of frequency and percentage distribution. Descriptive statistics were calculated based on health economic endpoints. Fisher's Exact test was used for statistical comparison of categorical variables.

Statistical analysis was conducted using $S A S$ version 9.2 (SAS Institute, Cary, NC, USA). The study protocol was reviewed and approved by both local and national institutional review boards. The Institutional Review Board (IRB) of the Azienda ospedaliero-universitaria "Maggiore della Carità" in Novara (Principal Investigator: Prof. Gianni Bona) first approved the study, then all the IRBs of the participating centers were notified according to the regulatory laws at the time.

\section{Results}

A total of 4536 patients were screened and 944 subjects with high fever were selected for study inclusion between May 2008 and June 2009. The majority of screening failures had a missing reason or 'other' reason for non- 
enrolment (Fig. 1). After exclusion of eight children due to age greater than five years, two due to a lack of an analyzed blood sample, thirteen due to not being a resident for at least three months and one patient for whom only demographic data were collected, 920 children were enrolled in the study. The mean age at enrolment was 23.1 months (range 0-59), and $58 \%$ (529/920) of the children were male. The age group with the greatest proportion of enrolled children was the one year of age group (Table 1, Fig. 2). Among the 814 children with a nonrectal temperature measurement from home, the mean temperature was $39.5{ }^{\circ} \mathrm{C}$; among the 97 children with a rectal temperature home measurement the mean temperature was $39.7{ }^{\circ} \mathrm{C}$. Home temperature was not recorded in nine cases. The mean temperature at enrolment was $38.7{ }^{\circ} \mathrm{C}$ (range 34.7-41.1), and $73 \%$ (672/920) of children with fever were hospitalized. At least one predisposing factor was present for $1.4 \%$ (13/920) of children (e.g. oncologic disease, immunodeficiency, intracatheter or central shunt, congenital cardiac anomaly). Thirty-seven percent (339/920) of children reported having received at least one dose of the 7-valent pneumococcal conjugate vaccine (PCV7; Prevenar, Pfizer/Wyeth). Antibiotic use before enrolment was reported for $42 \%$ (389/920) of children, and antibiotic use for greater than $24 \mathrm{~h}$ was reported for $29 \%(262 / 920)$ of children. Antipyretic treatment was reported for $88 \%(812 / 920)$ of children. Systemic inflammatory response syndrome (SIRS) [12] was present in $55 \%(507 / 920)$ of study patients.
A total of 225 children had a clinical diagnosis of ID, 38 had a diagnosis of sepsis, and 629 had a clinical diagnosis of non-invasive disease (Fig. 1). Thirty-four of the 920 children (3.7 \%) were considered bacteremic cases, having at least one positive sample, detected either by molecular assessment (PCR), culture, or both. Among children with a clinical diagnosis of ID, $9.8 \%(22 / 225)$ were bacteremic and among children with sepsis, $5.3 \%$ $(2 / 38)$ were bacteremic, whereas $1.6 \%$ (10/629) of children with a clinical diagnosis of non-invasive disease were bacteremic. PCR was carried out on all the samples, reaching $91 \%(31 / 34)$ positivity. Culture analysis was performed on $25 / 34$ samples; $36 \%(9 / 25)$ were positive. Of note, one of the culture-positive samples yielded Escherichia Coli, which was not targeted for detection with the PCR. On samples tested simultaneously with both RT-PCR and cultures, $72 \%$ of cases were diagnosed by RT-PCR alone. The overall underestimation factor associated with the use of culture only was 2.53 .

The most common diagnosis was CAP, diagnosed in $44.1 \%(15 / 34)$ of bacteremic cases and $22.5 \%(199 / 886)$ of non-bacteremic cases $(p=0.003)$ (Table 1). Pleural effusion was diagnosed in $11.8 \%(4 / 34)$ of bacteremic and $0.5 \%(4 / 886)$ of non-bacteremic cases $(p<0.0001)$ and in $4 / 15$ of CAP (26.7\%). Meningitis was diagnosed in $11.8 \%(4 / 34)$ of bacteremic and $0.2 \%(2 / 886)$ of nonbacteremic children $(p<0.0001)$.

$S$. pneumoniae was the most frequently detected bacteria among bacteremic cases, accounting for $85.3 \%$

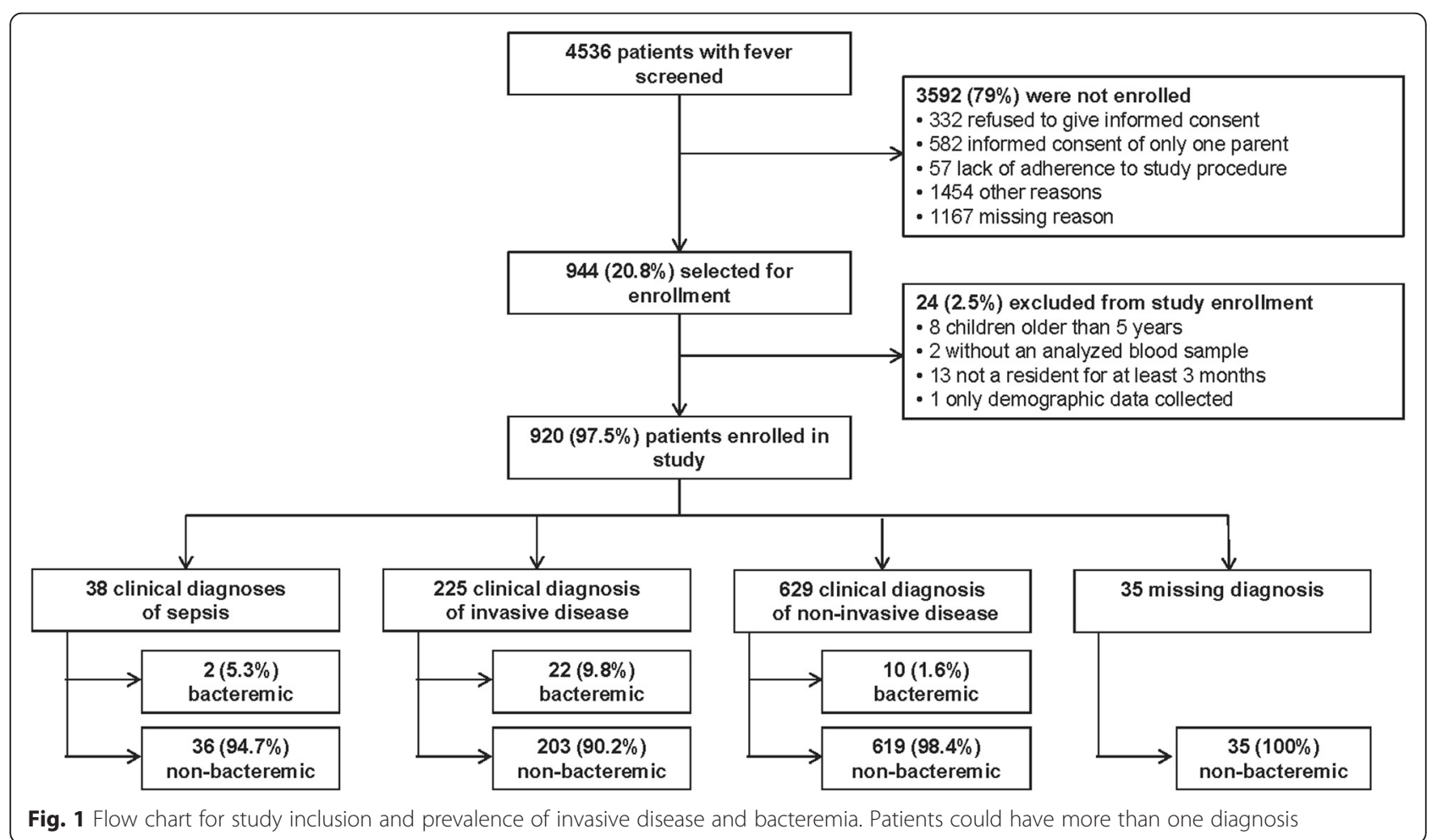

Fig. 1 Flow chart for study inclusion and prevalence of invasive disease and bacteremia. Patients could have more than one diagnosis 
Table 1 Characteristics and clinical diagnoses of bacteremic and non-bacteremic cases

\begin{tabular}{|c|c|c|c|}
\hline & Bacteremic $(N=34)$ & Non-bacteremic $(N=886)$ & All cases $(N=920)$ \\
\hline \multicolumn{4}{|l|}{ Age } \\
\hline$<1$ year & $9(26.5 \%)$ & $242(27.3 \%)$ & $251(27.3 \%)$ \\
\hline 1 year & $7(20.6 \%)$ & $273(30.8 \%)$ & $280(30.4 \%)$ \\
\hline 2 years & $8(23.5 \%)$ & $159(17.9 \%)$ & $167(18.2 \%)$ \\
\hline 3 years & $7(20.6 \%)$ & $131(14.8 \%)$ & $138(15.0 \%)$ \\
\hline 4 years & $3(8.8 \%)$ & $81(9.1 \%)$ & $84(9.1 \%)$ \\
\hline \multicolumn{4}{|l|}{ Prior antibiotic treatment } \\
\hline No & $17(50.0 \%)$ & $481(54.3 \%)$ & $498(54.1 \%)$ \\
\hline Yes & 16 (47.1\%) & $373(42.1 \%)$ & $389(42.3 \%)$ \\
\hline Unknown & $1(2.9 \%)$ & $32(3.6 \%)$ & $33(3.6 \%)$ \\
\hline \multicolumn{4}{|l|}{ Prior antipyretic treatment } \\
\hline No & $4(11.8 \%)$ & $79(8.9 \%)$ & $83(9.0 \%)$ \\
\hline Yes & 30 (88.2 \%) & $782(88.3 \%)$ & $812(88.3 \%)$ \\
\hline Unknown & 0 & $25(2.8 \%)$ & $25(2.7 \%)$ \\
\hline \multicolumn{4}{|l|}{ Temperature at enrolment (axillary) } \\
\hline$<39^{\circ} \mathrm{C}$ & $18(52.9 \%)$ & $452(51.0 \%)$ & $470(51.1 \%)$ \\
\hline $39-39.4^{\circ} \mathrm{C}$ & $10(29.4 \%)$ & $239(27.0 \%)$ & 249 (27.1\%) \\
\hline $39.5-39.9^{\circ} \mathrm{C}$ & $6(17.6 \%)$ & $130(14.7 \%)$ & 136 (14.8 \%) \\
\hline$\geq 40^{\circ} \mathrm{C}$ & 0 & $63(7.1 \%)$ & $63(6.8 \%)$ \\
\hline Missing & 0 & $2(0.2 \%)$ & $2(0.2 \%)$ \\
\hline \multicolumn{4}{|l|}{ Clinical diagnosis of invasive disease $\mathrm{e}^{\mathrm{a}}$} \\
\hline Community-acquired pneumonia & $15(44.1 \%)$ & $199(22.5 \%)$ & $214(23.3 \%)$ \\
\hline Pleural effusion & $4(11.8 \%)$ & $4(0.5 \%)$ & $8(0.9 \%)$ \\
\hline Meningitis & $4(11.8 \%)$ & $2(0.2 \%)$ & $6(0.7 \%)$ \\
\hline Sepsis & $2(5.9 \%)$ & $36(4.1 \%)$ & $38(4.1 \%)$ \\
\hline \multicolumn{4}{|c|}{ Clinical diagnosis of non-invasive disease ${ }^{a}$} \\
\hline Cellulites & 1 (2.9\%) & $5(0.6 \%)$ & $6(0.7 \%)$ \\
\hline Lymphoadenitis & 0 & $12(1.4 \%)$ & $12(1.3 \%)$ \\
\hline Otitis media & $3(8.8 \%)$ & $38(4.3 \%)$ & $41(4.5 \%)$ \\
\hline Pharyngitis & 0 & $27(3.0 \%)$ & $27(2.9 \%)$ \\
\hline Genitourinary infection & $1(2.9 \%)$ & $45(5.1 \%)$ & $46(5.0 \%)$ \\
\hline Other infection & $5(14.7 \%)$ & $500(56.4 \%)$ & $505(54.9 \%)$ \\
\hline
\end{tabular}

${ }^{a}$ Cases could have more than one primary and/or secondary diagnosis

(29/34) of cases. H. influenzae was detected in three cases (2 non-typeable and 1 capsulated), Escherichia Coli in one, and Neisseria meningitidis in one (Table 2). The most commonly detected $S$. pneumoniae serotype was 19A, detected in four cases. Among the 15 cases of CAP, 14 were due to $S$. pneumoniae, with three due to serotype 3 and three due to serotype 14. All four cases of pleural effusion were due to $S$. pneumoniae, while three of the four cases of meningitis were due to S. pneumoniae with the remaining case was due to Neisseria meningitidis. Serotypes are shown in Table 2. Among enrolled cases with oropharyngeal swab results available, $47.6 \%(429 / 902)$ were colonized by a various mix of the studied bacterial pathogens; $90 \%$ (27/30) of bacteremic cases were colonized compared to $46.1 \%(402 / 872)$ of non-bacteremic cases $(p<0.001)$.

From parents' recollection, PCV7 vaccination was reported for $62.1 \%(18 / 29)$ of bacteremic cases caused by S. pneumoniae. Six of the 18 S. pneumoniae cases reportedly received three doses of the vaccine (cases were due to serotypes 3,14, 18, 19A and non-typeable (two)). As for the case due to serotype 18 , the laboratory analysis method used could not differentiate serotype 18B (nonvaccine serotype) from $18 \mathrm{C}$ (vaccine serotype). Another six reportedly received two doses (cases were due to serotypes 1 (two), 7F-7A, 14, non-typeable and one missing). As 


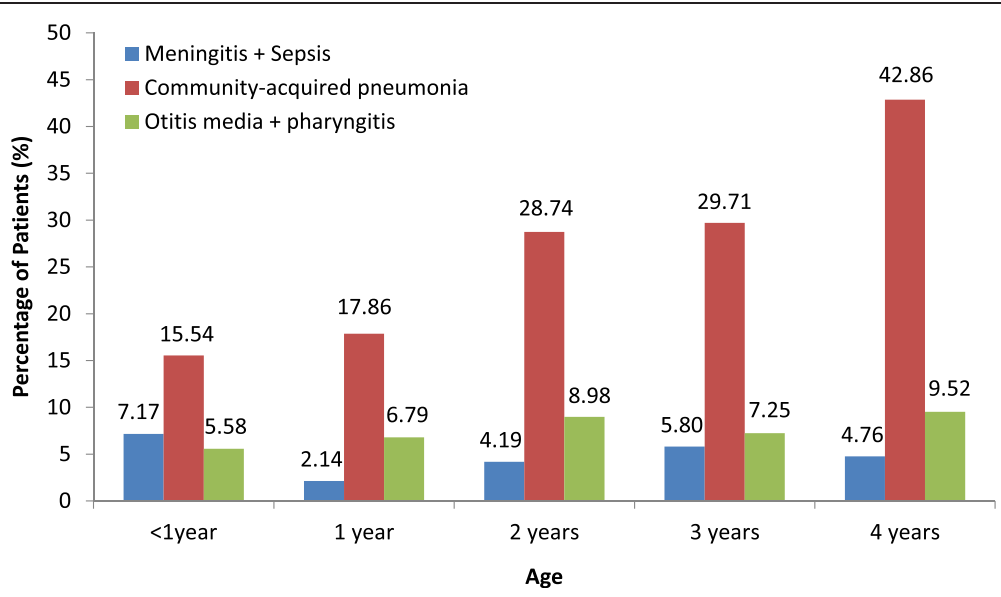

Fig. 2 Distribution of confirmed diagnoses by age among the enrolled children

for the IPD caused by serotype 14, the two doses had been administered at the fourth and seventh month of life, while the dose that is recommended in the second year of life had not been administered. The remaining six reportedly received one dose (cases were due to serotypes 3, 7F-7A, 19F (two) and non-typeable (two)).

The health economics study included 174 of the enrolled children. The mean duration of hospitalization was 6.47 days (range 1-38) and was longer for bacteremic
(13.6 days, standard deviation 9.6) than non-bacteremic (5.8 days, standard deviation 4.0) cases. The mean number of working hours lost was 28.5 (standard deviation 32.4), and was higher for the two bacteremic cases with data available (108, standard deviation 45.3) than for the 75 non-bacteremic cases with data available (26.3, standard deviation 29.6). The mean costs sustained by the family were 107.4 euro (standard deviation 347.6), with a mean cost of 1300 euro (standard deviation 1838) for the two

Table 2 Distribution of bacteria and serotypes for the 34 bacteremic cases by clinical diagnosis

\begin{tabular}{|c|c|c|c|c|c|c|c|c|c|}
\hline $\begin{array}{l}\text { Bacterium and } \\
\text { serotype }\end{array}$ & $\begin{array}{l}\text { Community-acquired } \\
\text { pneumonia }(N=15)\end{array}$ & $\begin{array}{l}\text { Pleural effusion } \\
(N=4)\end{array}$ & $\begin{array}{l}\text { Meningitis } \\
(N=4)\end{array}$ & $\begin{array}{l}\text { Sepsis } \\
(N=2)\end{array}$ & $\begin{array}{l}\text { Cellulites } \\
(N=1)\end{array}$ & $\begin{array}{l}\text { Otitis media } \\
(N=3)\end{array}$ & $\begin{array}{l}\text { Genitourinary } \\
\text { infection }(N=1)\end{array}$ & $\begin{array}{l}\text { Other infection } \\
(N=5)\end{array}$ & $\begin{array}{l}\text { Total } \\
(N=34)\end{array}$ \\
\hline Escherichia coli & & & & & & & & 1 (2.9\%) & 1 (2.9\%) \\
\hline $\begin{array}{l}\text { Neisseria } \\
\text { meningitidis C }\end{array}$ & & & 1 (2.9\%) & & & & & & 1 (2.9\%) \\
\hline $\begin{array}{l}\text { Haemophilus } \\
\text { influenzae (total) }\end{array}$ & 1 (2.9\%) & & & & & & & $2(5.9 \%)$ & $3(8.8 \%)$ \\
\hline Non-typeable & 1 (2.9\%) & & & & & & & 1 (2.9\%) & $2(5.9 \%)$ \\
\hline Serotype missing & & & & & & & & 1 (2.9 \%) & 1 (2.9 \%) \\
\hline $\begin{array}{l}\text { Streptococcus } \\
\text { pneumoniae (total) }\end{array}$ & $14(41.2 \%)$ & $4(11.8 \%)$ & $3(8.8 \%)$ & $2(5.9 \%)$ & $1(2.9 \%)$ & $3(8.8 \%)$ & & $3(8.8 \%)$ & $29(85.3 \%)$ \\
\hline Serotype 1 & 2 (5.9 \%) & & & & & & & & 2 (5.9\%) \\
\hline Serotype $3^{a}$ & $3(8.8 \%)$ & 1 (2.9\%) & & & & & & & 3 (8.8 \%) \\
\hline Serotype 5 & 1 (2.9 \%) & 1 (2.9\%) & & & & & & & 2 (5.9\%) \\
\hline Serotype 6A & & & & & & 1 (2.9\%) & & & 1 (2.9\%) \\
\hline Serotype 7F-7A & & 1 (2.9\%) & 1 (2.9\%) & & & & & & 2 (5.9\%) \\
\hline Serotype 14 & 3 (8.8 \%) & & & & & & & & 3 (8.8 \%) \\
\hline Serotype 18 & 1 (2.9 \%) & & & & & & & & 1 (2.9 \%) \\
\hline Serotype 19A & 1 (2.9\%) & 1 (2.9\%) & & 1 (2.9\%) & & & & 1 (2.9\%) & $4(11.8 \%)$ \\
\hline Serotype 19F & & & & & $1(2.9 \%)$ & 1 (2.9 \%) & & & 2 (5.9\%) \\
\hline Serotype 23F & & & 1 (2.9 \%) & & & & & & 1 (2.9\%) \\
\hline Non-typable & 2 (5.9 \%) & & 1 (2.9 \%) & & & 1 (2.9 \%) & & 1 (2.9\%) & $5(14.7 \%)$ \\
\hline Serotype missing & 1 (2.9\%) & & & 1 (2.9 \%) & & & & 1 (2.9 \%) & $3(8.8 \%)$ \\
\hline
\end{tabular}

${ }^{\mathrm{a}}$ One patient had community-acquired pneumonia and pleural effusion with Streptococcus pneumoniae 3 
bacteremic cases and 81.2 euro (standard deviation 231.6) for the 91 non-bacteremic cases. As there were only two bacteremic cases with data available on working hours lost and medical costs, comparisons between bacteremic and non-bacteremic cases could not be made.

\section{Discussion}

This study provides much needed information on the burden of bacteremia and ID among children less than five years of age with fever $\geq 39^{\circ} \mathrm{C}$ seeking hospital care in Italy. Thirty-four bacteremic cases out of 920 children with high fever were detected through blood assays; blood analysis (molecular or cultural method) is important for bacteremia diagnosis and should be implemented in children represented by our study population. The proportion of children with bacteremia is likely an underestimation of what is expected in the entire population, since not all patients present at the hospital, and not all presenting patients were enrolled in the study.

CAP and upper respiratory tract infections were the most common infections and reasons for hospitalizations. More than $50 \%$ of enrolled children presented with SIRS (used to measure severity of children seen in hospital), which confirms that this population is clinically relevant. A high proportion of children with fever were hospitalized, however, it should be noted that children presenting at the hospital with fever but with a good clinical profiles would not have been hospitalized and a blood sample would not be drawn, making the child ineligible for the study. Therefore, this figure is likely an overestimation.

S. pneumoniae was the most commonly detected (85\%) bacteria under study in bacteremic patients, followed by $H$. influenzae (9\%). Several of the bacteremic cases caused by S. pneumoniae, including cases due to PCV7 serotypes, reported having received at least one PCV7 dose. This finding was unexpected, and more detailed information on the timing of vaccination and underlying health of these children are necessary to better understand this finding. Two out of three $H$. influenzae cases were due to non-typeable $H$. influenzae, an emerging pathogen which causes invasive and respiratory infections; a preventive strategy should include as many pneumococcal serotypes as possible and hopefully, in the future, other bacteria causing bacteremia and ID.

Use of the newer molecular techniques for detection of bacteremia is a recent development, and there are very few studies on bacteremia in children in Italy using these techniques [10, 13]. Molecular methods for diagnosing and serotyping bacteremia, such as PCR, do not require viable bacteria, require smaller sample volumes than more traditional blood culture methods, and appear to be more sensitive $[10,11,13]$, allowing for a more accurate assessment of the burden of bacteremia. A highly sensitive technique is particularly necessary in the setting of antibiotic use, such as in our study population, where $42 \%$ of children had been exposed to antibiotics. Our results support the higher sensitivity of the molecular techniques versus the blood culture techniques.

Costs generated by a case of bacteremia can be consistent both in the National Healthcare System and in the families' perspective, with an estimated average direct medical cost of 3306 euro for each hospitalized case (range 511-19,418) based on the estimated daily hospitalization cost of 511 euro [14]. There is a high social cost of more than 100 euro/family on average, and hours lost from work, data which highlight the burden of disease on families of affected children.

A primary limitation of this study was the lower than expected sample size. Despite efforts to take the crowded nature and often overloaded healthcare personnel of the emergency departments into consideration in the study design, the target enrolment was still not met. The study aimed to recruit 4000 patients, but ultimately 920 were enrolled. The limited availability of data on reasons for non-enrolment makes it difficult to determine the primary obstacles to meeting target enrolment.

\section{Conclusions}

S. pneumoniae was confirmed to be one of the more frequent pathogen responsible for bacteremia, with over $80 \%$ of cases attributable to the pathogen. The study confirms the higher sensitivity of molecular versus cultural techniques for detecting bacteremia. Overall the study contributes to our understanding of the burden of IPD among Italian children, and provides important data on bacteremia and ID associated with high fever in this population. The study also documents the estimate of the costs associated with bacteremia and sustained by the families. Prevention of bacteremia by means of vaccination is thus warranted.

\section{Abbreviations \\ CAP: Community-acquired pneumonia; H. influenzae: Haemophilus influenzae; Hib: Haemophilus influenzae type b; ID: Invasive disease; IPD: Invasive pneumococcal disease; PCR: Polymerase chain reaction; PCV7: 7-valent pneumococcal conjugate vaccine; RT-PCR: Realtime polymerase chain reaction; S. pneumoniae: Streptococcus pneumoniae; SIRS: Systemic inflammatory response syndrome.}

\section{Competing interests}

FM and KH are employees of the GSK group of companies and hold stock options/shares. CA and MR reports payments from the GSK group of companies for support of travel to meetings for the study or other purposes. MM reports payments received by her institution for patents, royalties and development of educational presentations, and to her for royalties and development of educational presentations. PDP reports payments from the GSK group of companies for consultancy, expert testimony and from the GSK group of companies to his institution for grants. CDB reports a payment received by its institution from the GSK group of companies for a grant. SE reports payment from Novartis for board membership, Crucell, GSK group of companies, Pfizer, Novartis for lectures including service on speakers bureau and payment made to her institution from Crucell, Roche, GSK group of 
companies, Pfizer, Novartis for grant, from Novartis for board membership. LC is an employee of CROS NT which received fees from the GSK group of companies for participating in reviews activities such as data monitoring boards, statistical analysis, end point committee, and the like. FM, GC-G, AC, $\mathrm{FdB}$ and $\mathrm{GB}$ report no conflict of interest.

\section{Authors' contributions}

All authors served as principal investigators and/or were involved in the original idea and design of the study, wrote the protocol and report, or analyzed and interpreted data. All authors reviewed and approved the manuscript. All authors had full access to the data and were involved in the drafting of the article or revising it critically for important intellectual content, and final approval of the manuscript. The corresponding author had final responsibility to submit for publication.

\section{Acknowledgements}

GlaxoSmithKline Biologicals SA was the funding source and was involved in all stages of the study conduct and analysis. GlaxoSmithKline Biologicals SA also funded all costs associated with the development and the publishing of the present manuscript. All authors had full access to the data and agreed with the submission of the publication. The authors would like to thank Roeland Van Kerckhoven (Keyrus Biopharma on behalf of GSK Vaccines), Jérémie Dedessus le Moutier and Grégory Leroux (both Business and Decision Life Sciences on behalf of GSK Vaccines) for manuscript coordination and Anna Dow (Freelance) for writing support.

\section{Trademark}

Prevenar is a registered trademark of Pfizer/Wyeth, USA.

\section{Author details}

${ }^{1}$ Division of Pediatric Immunology, Department of Health Sciences, University of Florence and Anna Meyer Children's University Hospital, Florence, Italy. ${ }^{2}$ Istituto Giannina Gaslini, Ospedale Pediatrico IRCCS, Via Gerolamo Gaslini 5, 16148 Genoa, Italy. ${ }^{3}$ Azienda Ospedaliera Universitaria, Policlinico Consorziale di Bari, Piazza Giulio Cesare 11, 70124 Bari, Italy. ${ }^{4}$ Anna Meyer Children's University Hospital, Florence, Italy. ${ }^{5}$ Pediatric Highly Intensive Care Unit, Università degli Studi di Milano, Fondazione IRCCS Ca' Granda Ospedale Maggiore Policlinico, Milan, Italy. ${ }^{6}$ Paediatric Division, Bambino Gesù Children Hospital, v. Aurelia Km.30, 00100 Palidoro, Rome, Italy. ${ }^{7}$ Ospedale Santobono, Via Fiore Mario 6, 80129 Naples, Italy. ${ }^{8}$ Ospedale Gaspare Salesi, via F. Corridoni, 60120 Ancona, Italy. 'Azienda Ospedaliera Maggiore della Carita', Corso Mazzini, n. 18, Novara 28100, Italy. ${ }^{10}$ CROS NT, SRL, Via Germania 2, 37136 Verona, Italy. ${ }^{11}$ GSK Vaccines, Avenue Fleming 3, 1300 Wavre, Belgium. ${ }^{12}$ GSK Vaccines, Via A. Fleming 2, 37135 Verona, Italy.

Received: 28 February 2014 Accepted: 12 October 2015

Published online: 20 November 2015

\section{References}

1. D'Ancona F, Alfonsi V, Caporali M, Ranghiasci A, Atti C d, on behalf of the Regional referents for infectious diseases and vaccinations. Pneumococcal conjugate, meningococcal $C$ and varicella vaccination in Italy. Eurosurveillance. 2007:12:2

2. Whitney CG, Farley MM, Hadler J, Harrison LH, Bennett NM, Lynfield R, et al Decline in invasive pneumococcal disease after the introduction of proteinpolysaccharide conjugate vaccine. N Engl J Med. 2003;348:1737-46.

3. Ansaldi F, de Florentis D, Canepa P, Bandettini R, Diana MC, Martini M, et al. Epidemiological changes after PCV7 implementation in Italy: perspective for new vaccines. Hum Vaccin. 2011;7(Suppl):211-6.

4. Rodenburg GD, de Greeff SC, Jansen AG, de Melker HE, Schouls LM, Hak E, et al. Effects of pneumococcal conjugate vaccine 2 years after its introduction, the Netherlands. Emerg Infect Dis. 2010;16:816-23.

5. Kyaw MH, Lynfield R, Schaffner W, Craig AS, Hadler J, Reingold A, et al. Effect of introduction of the pneumococcal conjugate vaccine on drug-resistant Streptococcus pneumoniae. N Engl J Med. 2006;354:1455-63.

6. Azzari C, Canessa C, Lippi F, Moriondo M, Indolfi G, Nieddu F, et al. Distribution of invasive meningococcal $B$ disease in Italian pediatric population: implications for vaccination timing. Vaccine. 2014;32:1187-91.

7. D'Ancona F, Salmaso S, Barale A, Boccia D, Lopalco PL, Rizzo C, et al. Incidence of vaccine preventable pneumococcal invasive infections and blood culture practices in Italy. Vaccine. 2005;23:2494-500.
8. Pantosti A, Boccia D, D'Ambrosio F, Recchia S, Orefici G, Moro ML, et al. Inferring the potential success of pneumococcal vaccination in Italy: serotypes and antibiotic resistance of Streptococcus pneumoniae isolates from invasive diseases. Microb Drug Resist. 2003;9 Suppl 1:S61-8.

9. Resti M, Micheli A, Moriondo M, Becciolini L, Cortimiglia M, Canessa C, et al. Comparison of the effect of antibiotic treatment on the possibility of diagnosing invasive pneumococcal disease by culture or molecular methods: a prospective, observational study of children and adolescents with proven pneumococcal infection. Clin Ther. 2009;31:1266-73.

10. Azzari C, Moriondo M, Indolfi G, Cortimiglia M, Canessa C, Becciolini L, et al. Realtime PCR is more sensitive than multiplex PCR for diagnosis and serotyping in children with culture negative pneumococcal invasive disease. PLoS One. 2010;5, e9282.

11. Resti M, Moriondo M, Cortimiglia M, Indolfi G, Canessa C, Becciolini L, et al. Community-acquired bacteremic pneumococcal pneumonia in children: diagnosis and serotyping by real-time polymerase chain reaction using blood samples. Clin Infect Dis. 2010;51:1042-9.

12. Goldstein B, Giroir B, Randolph A. International Consensus Conference on Pediatric Sepsis. International pediatric sepsis consensus conference: definitions for sepsis and organ dysfunction in pediatrics. Pediatr Crit Care Med. 2005;6:2-8

13. Azzari $C$, Moriondo M, Indolfi G, Massai C, Becciolini $L$, de Martino $M$, et al. Molecular detection methods and serotyping performed directly on clinical samples improve diagnostic sensitivity and reveal increased incidence of invasive disease by Streptococcus pneumoniae in Italian children. J Med Microbiol. 2008;57:1205-12.

14. Azzari C, Massai C, Poggiolesi C, Indolfi G, Spagnolo G, De Luca M, et al. Cost of varicella-related hospitalisations in an Italian paediatric hospital: comparison with possible vaccination expenses. Curr Med Res Opin. 2007;23:2945-54

\section{Submit your next manuscript to BioMed Central and take full advantage of:}

- Convenient online submission

- Thorough peer review

- No space constraints or color figure charges

- Immediate publication on acceptance

- Inclusion in PubMed, CAS, Scopus and Google Scholar

- Research which is freely available for redistribution 